\title{
A sub-kiloparsec-scale view of un-lensed submillimeter galaxies
}

\author{
Ken-ichi Tadaki ${ }^{1}(\mathbb{D})$ and Daisuke Iono ${ }^{1,2}$ \\ ${ }^{1}$ National Astronomical Observatory of Japan, 2-21-1 Osawa, Mitaka, Tokyo 181-8588, Japan \\ email: tadaki.ken@nao.ac.jp \\ ${ }^{2}$ Department of Astronomical Science, SOKENDAI (The Graduate University for \\ Advanced Studies), Mitaka, Tokyo 181-8588, Japan
}

\begin{abstract}
Submillimeter galaxies at $z>3$ building up their central cores through compact starbursts with an effective radius of 1-2 kpc. Our ALMA high-resolution observations reveal off-center gas clumps in a submillimeter galaxy at $z=4.3$, COSMOS-AzTEC-1, as well as a rotation-dominated disk. Exploiting the kinematic properties and the spatial distribution of gas mass surface density, we find that the starburst disk is gravitationally unstable. This result is consistent with a scenario where in-situ clumps are formed through disk instability. On the other hand, we find evidence for an ex-situ clump that does not corotate with the starburst disk. The accretion of such a non-corotating clump could stimulate violent disk instability, driving gas inflows into the central regions of the galaxy. Our results suggest that compact cores are formed through an extreme starburst due to a gravitational instability, triggered by non-corotating clumps.
\end{abstract}

Keywords. galaxies: formation, galaxies: starburst, galaxies: ISM

\section{Introduction}

When and how did galaxies shape the Hubble sequence? Over the past three decades, high-resolution, high-quality images obtained with Hubble Space Telescope (HST) revealed the rest-optical morphologies for tens of thousands of distant galaxies. The most striking results are

1) a correlation between star-forming activity and morphology of galaxies at $0<z<2.5$ : star-forming galaxies are typically a disk-dominated system whereas quiescent galaxies are a spheroid-dominated one (e.g., Wuyts et al. 2011; Bell et al. 2012),

2) a discovery of massive, compact quiescent galaxies at $z \sim 2$ : the half-light radius of $R_{1 / 2} \sim 1 \mathrm{kpc}$ is a factor of $4-5$ smaller than similar mass quiescent galaxies at $z=0$ (e.g., Trujillo et al. 2006; van Dokkum et al. 2015), and

3 ) size evolution from $z=3$ to $z=0$ : star-forming galaxies are always larger than quiescent galaxies and both galaxies gradually increase size over time (e.g., van der Wel et al. 2014).

These findings offered a paradigm for galaxy evolution: high-redshift massive galaxies evolve into giant ellipticals through two channels. In a fast channel, massive compact starforming galaxies will become compact quiescent galaxies at $z \sim 2$ after quenching star formation (e.g., Barro et al. 2013). Repeating minor mergers puffs up compact quiescent galaxies, turning them into giant elliptical galaxies (e.g., Naab et al. 2009). In a slow channel, galaxies increase their size by in-situ star formation, and after quenching larger quenched galaxies continuously add to the quiescent population at $z=0-1$ (e.g., Carrolo et al. 2013; Belli et al. 2015). This two-channel scenario explains the redshift evolution 


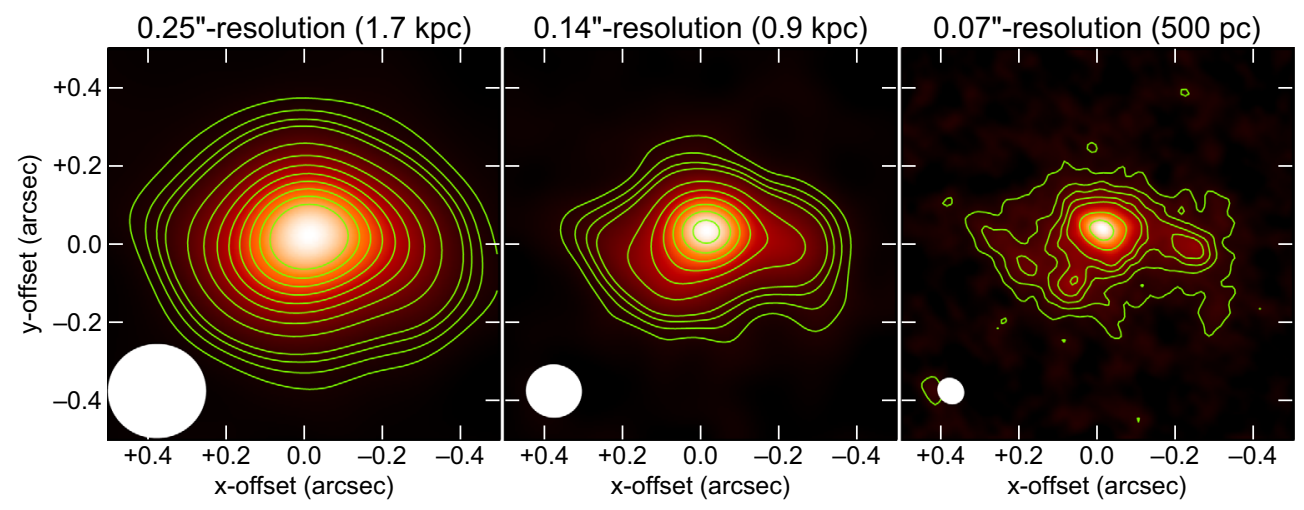

Figure 1. ALMA $870 \mu \mathrm{m}$ continuum maps at three different spatial resolutions in COSMOSAzTEC-1 at $z=4.3$ (Iono et al. 2016). The image size is $1^{\prime \prime} \times 1 .{ }^{\prime \prime}$ Contours are plotted every $3 \sigma$ from $6 \sigma$ to $15 \sigma$ and every $10 \sigma$ from $15 \sigma$.

of both the size and the number density of star-forming/quiescent galaxies (e.g., Barro et al. 2013; van Dokkum et al. 2015). However, a big problem still remains: how did compact star-forming galaxies form at $z=3-6$ ?

\section{Overview}

Submillimeter bright galaxies (SMGs) at $z>3$ are the most likely progenitors of compact star-forming galaxies. Recent $0 .^{\prime \prime} 2$-resolution dust continuum observations indicate that SMGs are vigorously forming stars in the central 1-2 kpc region, supporting an evolutionary link from SMGs to compact star-forming galaxies with $R_{1 / 2} \sim 1 \mathrm{kpc}$ (e.g., Simpson et al. 2015; Ikarashi et al. 2015; Tadaki et al. 2017). What happens if we go to higher resolution? ALMA 0."07-resolution observations of $870 \mu \mathrm{m}$ continuum reveal that a bright SMG at $z=4.3$, COSMOS-AzTEC-1, is not just compact, but also has clumpy structures in the central 1-2 kpc region (Figure 1; Iono et al. 2016). Such offcenter clumps are now commonly seen in SMGs (Hodge et al. 2019; Rujopakarn et al. 2019 although we need a better resolution than $0 .{ }^{\prime \prime} 1$ to prove them (Figure 1).

An obvious next step is to investigate the kinematics in the central starburst region to understand the origin of off-center clumps. Unfortunately, dust continuum observations do not provide information of kinematics in galaxies. We therefore obtained 0."08-resolution observation of the $\mathrm{CO}(4-3)$ line in COSMOS-AzTEC-1 (Tadaki et al. 2018). The starburst disk is rotation-dominated with a rotation velocity-to-velocity dispersion ratio of $v / \sigma_{0} \sim 3$ (Figure 2). The ordered rotation is also confirmed by [C II] and [N II] line observations (Figure 3; Tadaki et al. 2019a). The measured Toomre $Q$ parameter is much below unity over the starburst disk $\left(Q_{\text {obs }} \sim 0.2\right)$, suggesting that off-center clumps are formed through the gravitational instability in the central 1-2 kpc region.

On the other hand, we have discovered a non-corotating clump by $0 .{ }^{\prime \prime} 17$-resolution [C II] observations (Tadaki et al. 2019b, in prep). The [C II] clump has a large velocity offset of $\sim 200 \mathrm{~km} \mathrm{~s}^{-1}$ from the disk component and is located along the kinematic minor axis of disk rotation. We suggest a scenario where the ex-situ non-corotating clump develops the violent disk instability (VDI), where the disk is turbulent and highly perturbed, driving gas inflow into the central region of the galaxy (Dekel et al. 2009; Dekel \& Burkert 2014; Danovich et al. 2015; Zolotov et al. 2015). The extreme starburst is likely to be caused by a combination of minor mergers and efficient star formation due to gravitational instability in the central 1-2 kpc region. 

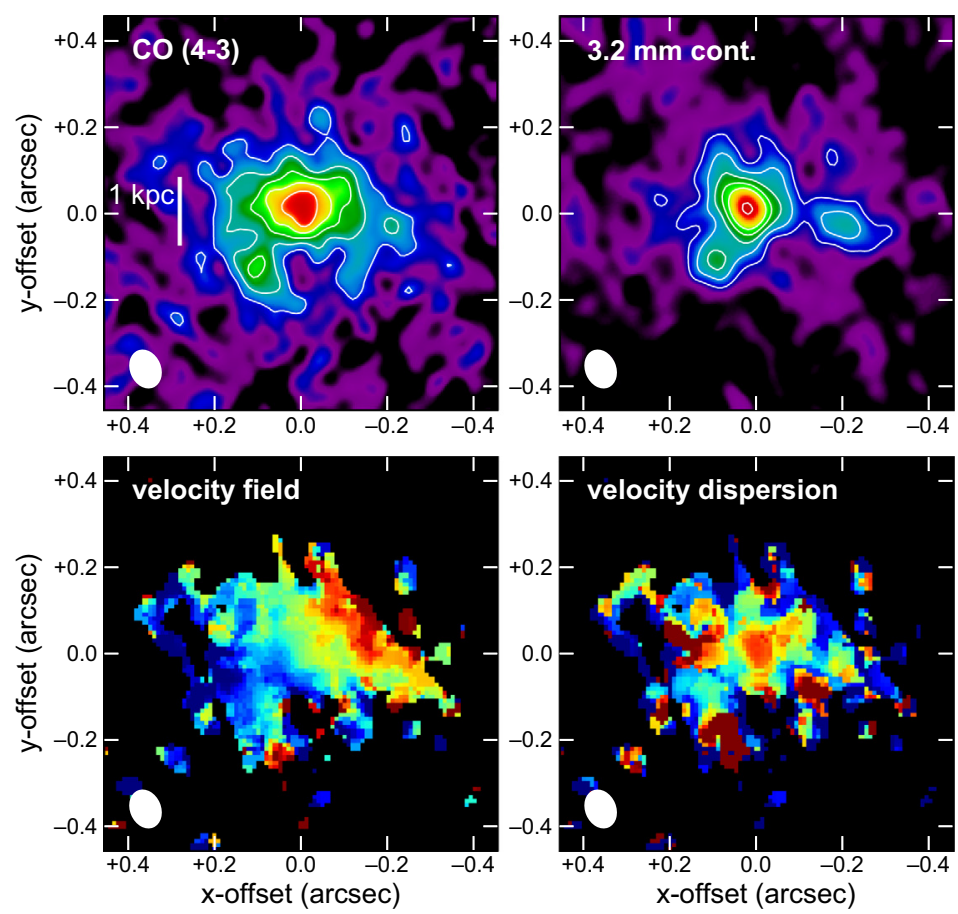

Figure 2. ALMA maps of the CO (4-3) line, $3.2 \mathrm{~mm}$ continuum, velocity field, velocity dispersion (Tadaki et al. 2018).

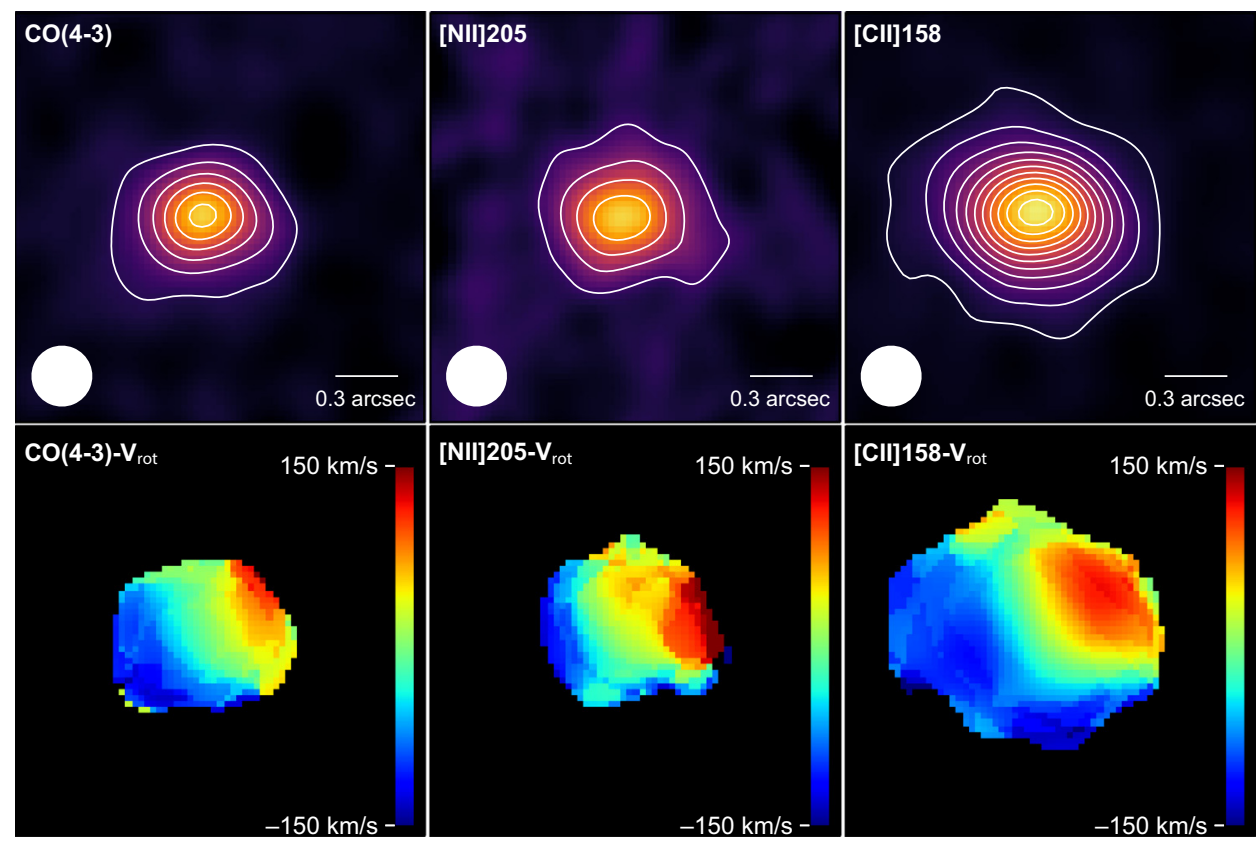

Figure 3. ALMA $\mathrm{CO}(4-3)$, $[\mathrm{NII}]$ and $[\mathrm{CII}]$ maps of flux and velocity field in COSMOSAzTEC-1 (Tadaki et al. 2019a). The spatial resolutions are all $\sim 0 .{ }^{\prime \prime} 3$. The image size is $2^{\prime \prime} \times 2$." Contours are plotted every $5 \sigma$ from $3 \sigma$. 
ALMA observations of molecular/ionized/PDR gas alone are not enough to reject the possibility of major mergers in COSMOS-AzTEC-1, because gas and new stars formed after mergers become rotation-dominated in both cases of major and minor mergers (Robertson et al. 2006). While COSMOS-AzTEC-1 is very faint at $<2 \mu \mathrm{m}$, it is sufficiently bright at $3-4 \mu \mathrm{m}$ with an $\mathrm{AB}$ magnitude of 22 . Integral field spectroscopy with Near Infrared Spectrograph on James Webb Space Telescope (Dorner et al. 2016) will allow us to investigate the stellar kinematics through observations of stellar absorption lines in the rest-frame optical wavelengths. The ALMA-JWST synergetic observations will enable us to determine whether SMGs experienced major mergers in the past and understand the physical mechanism responsible for the extreme starburst in the early Universe.

\section{References}

Barro, G., Faber, S. M., Pérez-González, P. G., et al. 2013, ApJ, 765, 104

Bell, E. F., van der Wel, A., Papovich, C., et al. 2012, ApJ, 753, 167

Belli, S., Newman, A. B., \& Ellis, R. S. 2015, ApJ, 799, 206

Carollo, C. M., Bschorr, T. J., Renzini, A., et al. 2013, ApJ, 773, 112

Danovich, M., Dekel, A., Hahn, O., Ceverino, D., \& Primack, J. 2015, MNRAS, 449, 2087

Dekel, A., Birnboim, Y., Engel, G., et al. 2009, Nature, 457, 451

Dorner, B., Giardino, G., Ferruit, P., et al. 2016, A\&AA, 592, A113

Dekel, A. \& Burkert, A. 2014, MNRAS, 438, 1870

Hodge, J. A., Smail, I., Walter, F., et al. 2019, ApJ, 876, 130

Ikarashi, S., Ivison, R. J., Caputi, K. I., et al. 2015, ApJ, 810, 133

Iono, D., Yun, M. S., Aretxaga, I., et al. 2016, ApJL, 829, L10

Naab, T., Johansson, P. H., \& Ostriker, J. P. 2009, ApJL, 699, L178

Robertson, B., Bullock, J. S., Cox, T. J., et al. 2006, ApJ, 645, 986

Rujopakarn, W., Daddi, E., Rieke, G. H., et al. 2019, arXiv e-prints, arXiv:1904.04507

Simpson, J. M., Smail, I., Swinbank, A. M., et al. 2015, ApJ, 799, 81

Tadaki, K.-i., Genzel, R., Kodama, T., et al. 2017, ApJ, 834, 135

Tadaki, K., Iono, D., Yun, M. S., et al. 2018, Nature, 560, 613

Tadaki, K.-i., Iono, D., Hatsukade, B., et al. 2019, ApJ, 876, 1

Trujillo, I., Feulner, G., Goranova, Y., et al. 2006, MNRAS, 373, L36

van der Wel, A., Franx, M., van Dokkum, P. G., et al. 2014, ApJ, 788, 28

van Dokkum, P. G., Nelson, E. J., Franx, M., et al. 2015, ApJ, 813, 23

Wuyts, S., Förster Schreiber, N. M., van der Wel, A., et al. 2011, ApJ, 742, 96

Zolotov, A., Dekel, A., Mandelker, N., et al. 2015, MNRAS, 450, 2327 